\title{
Some Remarks on Hypoelliptic Operators which are not Micro-hypoelliptic
}

\author{
Dedicated to Professor Shigetake Matsuura on his sixtieth birthday \\ By \\ Yoshinori Morimoto* and Tatsushi MorioKA**
}

\section{§1. Introduction}

In this note we give an example of hypoelliptic operators which are not micro-hypoelliptic. Non-micro-hypoellipticiy of the example arises from the oscillation of the coefficient with a zero of infinite order.

Let us consider the following semi-elliptic operator with infinite degeneracy:

$$
L=a\left(x, y, D_{x}\right)+g(x) b\left(x, y, D_{y}\right) \quad \text { in } \quad \mathbf{R}^{n}=\mathbf{R}_{x}^{n_{1}} \times \mathbf{R}_{y}^{n_{2}} .
$$

Here $g(x) \in C^{\star}$ and satisfies

$$
g(x)>0 \text { for } x \neq 0 \text { and } \partial_{x}^{\beta} g(0)=0 \text { for any } \beta .
$$

Here $a\left(x, y, D_{x}\right)$ and $b\left(x, y, D_{y}\right)$ are differential operators with $C^{x}$ coefficients of order $2 \ell$ and $2 m$. We assume that $a\left(x, y, D_{x}\right)$ and $b\left(x, y, D_{y}\right)$ are strongly elliptic with respect to $x$ and $y$, respectively, that is, for $C_{1}, C_{2}>0$

$$
\begin{aligned}
& \operatorname{Re} a(x, y, \xi) \geq C_{1}|\xi|^{2 t} \quad \text { and } \\
& \operatorname{Re} b(x, y, \eta) \geq C_{2}|\eta|^{2 m}
\end{aligned}
$$

hold if $|\xi|$ and $|\eta|$ are sufficiently large. In [3] the one of authors (T.M.) proved that the operator $L$ is hypoelliptic, i.e.

$$
\text { sing supp } u=\operatorname{sing} \operatorname{supp} L u \text { for } u \in \mathscr{D}^{\prime} \text {. }
$$

This ameliorates the old work [2] (c.f. Fediǐ [1]) of another author (Y.M.). Actually, in [2] the following condition was required to show (1.2) in case of $m \geq 2$ :

$$
\left\{\begin{array}{l}
\text { There exist constants } C \text { and } \sigma(0<\sigma<1 /\{2(m-\ell+m \ell)\}) \\
\text { such that }\left|\partial_{x}^{\beta} g(x)\right| \leq C g(x)^{1-\sigma|\beta|} \text { for }|\beta| \leq 2(m-\ell+m \ell)
\end{array}\right.
$$

Communicated by T. Kawai, May 10, 1991.

1991 Mathematics Subject Classifications: 35H05

* School of Mathematics, Yoshida College, Kyoto University, Kyoto 606-01, Japan.

** Department of Mathematics, Faculty of Sciences. Osaka University, Osaka 560, Japan. 
In the recent paper [4] the one of authors (T.M.) also has studied the microhypoellipticity of $L$ and has given the following theorem:

Theorem A. Let $z=\left(x_{0}, y_{0} ; \xi_{0}, \eta_{0}\right)$ be a point in $T^{*}\left(\mathbb{R}^{n}\right)\left(x_{0}, \xi_{0} \in \mathbb{R}^{n_{1}}\right.$ and $y_{0}, \eta_{0} \in \mathbf{R}^{n_{2}}$ ) with $\left|\eta_{0}\right| \neq 0$. Let $L$ be the operator (1.1) satisfying (A.1)-(A.3).

(i) In the case where $\ell \geq m, L$ is micro-hypoelliptic at $z$, that is, $z \notin \mathrm{WF}(\mathrm{Lu})$ implies $z \notin \mathrm{WF} u$

(ii) In the case where $\ell<m, L$ is still micro-hypoelliptic at $z$ if $g(x)$ satisfies the following condition:

$$
\left\{\begin{array}{l}
\text { There exist constants } C \text { and } \tau(0<\tau<1 /\{2(m-\ell)\}) \\
\text { such that }\left|\partial_{x}^{\beta} g(x)\right| \leq C g(x)^{1-\tau|\beta|} \text { for }|\beta| \leq 2(m-\ell)
\end{array}\right.
$$

We remark that Theorem $\mathrm{A}$ is valid in the case where $g(x)$ vanishes finitely at $x=0$. In this case, (A.4) implies $g(x)=o\left(|x|^{2(m-t)}\right)$. If $x \in \mathbb{R}^{1}$ and $g(x)=x^{2 k}$ for an integer $k>0$ then it follows from (A.4) that $k>m-\ell$. By ParentiRodino [5], it is known that if $0<k \leq m-\ell$, hypoelliptic operator $D_{x}^{2 t}+x^{2 k} D_{y}^{2 m}$ in $\mathbf{R}^{2}$ is not micro-hypoelliptic at $(0,0 ; 0,1) \in T^{*}\left(\mathbf{R}^{2}\right)$. The condition (A.4) is satisfied when $g(x)=\psi(x)^{k}$ for some integer $k>m-\ell$ and some $C^{x}$ function $\psi(x)$ with $\psi(x)>0$ for $x \neq 0$. This fact can be seen by noticing that $\psi^{\prime}(x)^{2} \leq$ Const. $\psi(x)$ near the origin. On the other hand, we see that for integer $k>0$

$$
g_{k}(x)=\exp (-1 /|x|) \sin ^{2 k} \pi /|x|+\exp \left(-1 /|x|^{2}\right)
$$

(cf. Remark 2 in [2, Section 1])

does not satisfies (A.4) if $k \leq m-\ell$. In fact, for $|\beta|=2 k$ and integer $j>0$ we have

$$
\partial_{x}^{\beta} g_{k}(x)=O\left(e^{-j}\right), g_{k}(x)=O\left(e^{-j^{2}}\right),|x|=1 / j, \text { as } j \rightarrow \infty .
$$

In order to consider the necessity of (A.4), we set

$$
L_{k}=a\left(x, D_{x}\right)+g_{k}(x) b\left(x, D_{y}\right) \text { in } \mathbf{R}_{x}^{1} \times \mathbb{R}_{y}^{n^{2}},
$$

where $a$ and $b$ satisfies (A.2) and (A.3), respectively (but they are independent of $y$ variable).

Theorem B. Let $\ell, m$ and $k$ be positive integers such that $m \geq \ell+2$ and $k \leq m-\ell-1$. If $g_{k}(x)$ is the function (1.3) with $x \in \mathbb{R}^{1}$ and if $L_{k}$ is the above operator then $L_{k}$ is not micro-hypoelliptic at $z=\left(0, y_{0} ; 0, \eta_{0}\right) \in T^{*}\left(\mathbb{R}_{x}^{1} \times \mathbb{R}_{y}^{\prime \prime 2}\right)$ with $\eta_{0} \neq 0$.

If (A.4)' denotes the condition (A.4) with $\tau$ replaced by $\tau^{\prime}\left(0<\tau^{\prime}<\right.$ $1 /\{2(m-\ell-1)\}$ then Theorem B shows that (A.4)' is necessary in general for $L$ to be micro-hypoelliptic. Unfortunately, in case of $m=\ell+1$ the theorem says 
nothing concerning the necessity of conditions like (A.4). In the next section we shall give the proof of Theorem B influenced by [5] though our method is a little different from the one there. To end Introduction authors wish to express their hearty gratitude to Professor N. Iwasaki for useful discussions.

\section{\$2. Proof of Theorem B}

For the sake of simplicity we shall prove Theorem $\mathrm{B}$ in case of $y \in \mathbf{R}^{1}\left(n_{2}=1\right)$, $y_{0}=0$ and $\eta_{0}=1$ since the proof in general case is similar. Throughout this section we assume that $m \geq \ell+2$. We construct a singular solution $u(x, y)$ in the form

$$
u(x, y)=\sum_{j=1}^{\infty} \eta_{j}^{-4} \exp \left(i \eta_{j} y\right) u_{j}(x)
$$

where $\eta_{j}=\exp \left\{j^{2} / 4 m\right\}$. We require that $u_{j}(x) \in C_{0}^{x}$ satisfies

$$
\begin{gathered}
\text { supp } u_{j} \subset\left\{\left|x-j^{-1}\right| \leq j^{-2} / 3\right\} \equiv \Omega_{j}, \\
\widehat{u}_{j}(0)>1 /\left(2 \eta_{j}^{1 / 2}\right)
\end{gathered}
$$

and

$$
\left|\widehat{u}_{j}(\xi)\right| \leq C_{1} \eta_{j},
$$

where $C_{1}>0$ is a constant independent of $j$. Hereafter we denote constants by $C_{k}(k=1,2, \ldots)$ and $c$. Note that

$$
L_{k} u=\sum_{j=1}^{\infty} \eta_{j}^{-4} \exp \left(i \eta_{j} y\right)\left\{a\left(x, D_{x}\right)+g_{k}(x) b\left(x, \eta_{j}\right)\right\} u_{j}(x) .
$$

Setting $f_{j}(x)=\left\{a\left(x, D_{x}\right)+g_{k}(x) b\left(x, \eta_{j}\right)\right\} u_{j}(x)$ we require that the Fourier transform of $f_{j}(x)$ satisfies with $C_{2}>0$ independent of $j$

$$
\left|\widehat{f}_{j}(\xi)\right| \leq C_{2} \eta_{j}^{-N_{j}} \quad \text { on } \quad\left\{|\xi| \leq \eta_{j} / 2\right\}
$$

where $N_{j} \rightarrow \infty(j \rightarrow \infty)$. Furthermore, with $C_{3}>0$ and $c>0$ independent of $j$ we require

$$
\left|\widehat{f}_{j}(\xi)\right| \leq C_{3}<\eta_{j}>^{c} \text { for all } \xi \in \mathbb{R}^{1} \text {. }
$$

Once we could obtain $u_{j}(x)$ (and $\left.f_{j}(x)\right)$ satisfying $(2.2)-(2.6) u(x, y)$ of the form (2.1) would be the desired singular solution. In fact, let $\varphi(x)$ be arbitrary $C_{0}^{x}$ function such that $\varphi=1$ in a neighborhood of the origin and $\widehat{\varphi}(0)=1$. The support of $u$, shrinks to $x=0$ when $j$ tend to $x$ and the sum of finite terms of the right hand side of (2.1) belongs to $C^{x}$. In considering the wave front set of $u(x, y)$ near the origin we may regard the Fourier transform of $\varphi(x) \varphi(y) u(x, y)$ as follows: 


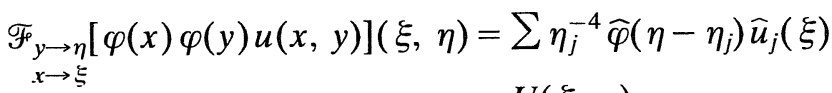

$$
\begin{aligned}
& \equiv U(\xi, \eta) \text {. }
\end{aligned}
$$

If $j \neq j^{\prime}$ we have

$$
\begin{aligned}
\left|\eta_{J}-\eta_{j^{\prime}}\right| & =\left|\eta_{j}^{1 / 2}-\eta_{j^{\prime}}^{1 / 2}\right|\left|\eta_{j}^{1 / 2}+\eta_{j^{\prime}}^{1 / 2}\right| \\
& \geq \max \left(\eta_{j}^{1 / 2}, \eta_{j^{\prime}}^{1 / 2}\right)
\end{aligned}
$$

because $\eta_{j}^{1 / 2}-\eta_{j-1}^{1 / 2}>1$. Write

$$
U\left(0, \eta_{j^{\prime}}\right)=\eta_{j^{\prime}}^{-4} \widehat{\varphi}(0) \widehat{u}_{j^{\prime}}(0)+\sum_{J \neq j^{\prime}} \eta_{J}^{-4} \widehat{\varphi}\left(\eta_{j^{\prime}}-\eta_{j}\right) \widehat{u}_{j}(0) .
$$

Since $\widehat{\varphi}(\eta) \in \mathscr{S}$ we have $|\widehat{\varphi}(\eta)| \leq C_{N}\left\langle\eta>^{-N}\right.$ for any integer $N$ and some constant $C_{N}$ the second term of the right hand side is majorated by $\eta_{j^{\prime}}^{-10}$ with a constant factor. By means of $(2.3)$ we have $U\left(0, \eta_{j^{\prime}}\right) \geq \eta_{j^{\prime}}^{-9 / 2} / 3\left(j^{\prime} \rightarrow \infty\right)$ and hence we see $(0,0 ; 0,1) \in \mathrm{WF} u$. On the other hand, we have

$$
V(\xi, \eta) \equiv \underset{\substack{\mathscr{F}^{\prime} \rightarrow \eta \\ x \rightarrow \xi}}{ }\left[\varphi(x) \varphi(y) L_{k} u\right](\xi, \eta)=\sum \eta_{J}^{-4} \widehat{\varphi}\left(\eta-\eta_{J}\right) \widehat{f}_{J}(\xi)
$$

For any fixed $\eta>0$, the terms with $j$ satisfying $\left|\eta^{1 / 2}-\eta_{j}^{1 / 2}\right| \geq 1$ are negligible because of (2.6). If there exists $j$ satisfying $\left|\eta^{1 / 2}-\eta_{j}^{1 / 2}\right|<1$ then it follows from (2.5) that $V(\xi, \eta)=0\left(\eta^{-N_{J}}\right)$ on $\{(\xi, \eta) ;|\xi| \leq \eta / 3\}$. Consequently, we see $(0,0$; $0,1) \notin \mathrm{WF} L_{k} u$.

Let us look for $u_{J}(x)$ and $f_{J}(x)$ satisfying (2.2)-(2.6). We shall consider the function $g_{k}(x)$ near $x=1 / j$. If $\alpha_{j}(x)=\int_{0}^{1} \rho(1 / j+(x-1 / j) \theta) d \theta$ with $\rho(t)=$ $\left(-1 / t^{2}\right) \cos \pi / t$ then $\sin \pi / x=\alpha_{j}(x)(x-1 / j)$ near $x=1 / j$ and hence

$$
g_{k}(x)=\beta_{j}(x)\left\{\left(x-j^{-1}\right)^{2 k}+\gamma_{j}(x)\right\} \quad \text { near } x=j^{-1},
$$

where $\beta_{j}(x)=\alpha_{j}(x)^{2 k} \exp (-1 / x)$ and $\gamma_{j}(x)=\alpha_{J}(x)^{-2 k} \exp \left\{1 / x-1 / x^{2}\right\}$. Note that

$$
\left|\beta_{j}(x)\right| \geq \exp (-2 j) \quad \text { in } \quad \Omega_{j}^{\prime} \equiv\left\{\left|x-j^{-1}\right| \leq j^{-2} / 2\right\}
$$

and for any integer $q>0$

$$
\begin{aligned}
\left|\beta_{j}^{(q)}(x)\right| & \leq C_{q} j^{4 k+2 q} \exp (-1 / x) \\
& \leq C_{q}^{\prime}\left(\log \eta_{j}\right)^{2 k+q} \exp (-1 / x) \text { in } \Omega_{j}^{\prime}
\end{aligned}
$$

hold with constants $C_{q}$ and $C_{q}^{\prime}$ independent of $j$. Here we used $\eta_{J}=\exp \left\{j^{2} / 4 m\right\}$. Similarly, we have

$$
\left|\gamma_{j}^{(q)}(x)\right| \leq C_{q}^{\prime \prime}\left(\log \eta_{j}\right)^{3 q / 2} \eta_{j}^{-2 m} \text { in } \Omega_{j}^{\prime} .
$$

Note that 


$$
\begin{aligned}
f_{j}(x)= & \left\{a\left(x, D_{x}\right)+g_{k}(x) b\left(x, \eta_{j}\right)\right\} u_{j}(x) \\
= & \beta_{j}(x) b\left(x, \eta_{j}\right) \eta_{j}^{-k}\left[\left\{\left(x-j^{-1}\right) \eta_{j}^{1 / 2}\right\}^{2 k}+\gamma_{j}(x) \eta_{j}^{k}\right. \\
& \left.+\beta_{j}(x)^{-1} b\left(x, \eta_{j}\right)^{-1} a\left(x, D_{x}\right) \eta_{j}^{k}\right] u_{j}(x) . \\
\equiv & \beta_{j}(x) b\left(x, \eta_{j}\right) \eta_{j}^{-k} \widetilde{f}_{j}(x) .
\end{aligned}
$$

Since (2.2) is required we may assume that $\beta_{j}(x)$ and $\gamma_{j}(x)$ belong to $C_{0}^{x}$ and satisfy $(2.8)-(2.9)$ in $(-\infty, \infty)$, by multiplying the cut function in $\Omega_{j}^{\prime}$ (equal to 1 on $\left.\Omega_{j}\right)$. If $\widehat{\beta}_{j}\left(\xi, \eta_{j}\right)$ denotes the Fourier transform of $\beta_{j}(x) b\left(x, \eta_{j}\right)$ then we have $\left|\widehat{\beta}_{j}\left(\xi, \eta_{j}\right)\right| \leq C_{q}\left(\log \eta_{j}\right)^{q+2 k} \eta_{J}^{2 m}<\xi>^{-q}$ for any $q>0$. Hence it suffices to require $\widetilde{f}_{j}(x)$ satisfies $(2.5)$ in $\left\{|\xi| \leq \eta_{j}\right\}$ and (2.6) instead of $f_{j}(x)$. In fact,

$$
\eta_{j}^{k} \widehat{f}_{j}(\xi)=\int \widehat{\beta}_{j}\left(\zeta-\xi, \eta_{j}\right) \widehat{\widetilde{f}}_{j}(\zeta) d \zeta=\int_{|\xi| \geq \eta_{j}} \cdot d \xi+\int_{|\zeta| \leqq \eta_{j}} \cdot d \zeta
$$

If $|\xi| \leq \eta_{J} / 2$ then the first term is estimated above from $C_{N_{j}}\left(\log \eta_{J}\right)^{N_{J}+2 k} \eta_{J}^{-N_{J}+2 m}$. The similar bound holds also for the second term because of $(2.5)$ for $\widetilde{f}_{j}(x)$.

Now we shall consider the equation

$$
\left[\left\{\left(x-j^{-1}\right) \eta_{i}^{1 / 2}\right\}^{2 k}+\gamma_{j}(x) \eta_{l}^{k}+\beta_{j}(x)^{-1} b\left(x, \eta_{j}\right)^{-1} a\left(x, D_{\imath}\right) \eta_{j}^{k}\right] u_{j}(x)=\widetilde{f}_{j}(x) .
$$

We shall omit the suffix $j$ for a while (by fixing $j$ ). If we write

$$
\gamma(x)+\beta(x)^{-1} b(x, \eta)^{-1} a\left(x, D_{x}\right)=\sum_{s=0}^{2 \ell} D_{r}^{s} c_{s}(x, \eta)
$$

by means of (2.7) $-(2.9)$ and (A.3) we see that for any $\varepsilon>0$

$$
\left|D_{x}^{q} c_{s}(x, \eta)\right| \leq C_{q, \varepsilon} \eta^{-2 m+\varepsilon}(\log \eta)^{3 q / 2} .
$$

Note that the left hand side of (2.10) equals

$$
\begin{aligned}
& \iint \exp -i\left\{\left(x-j^{-1}\right) \eta^{1 / 2} t-t \tau\right\} \times\left\{\tau^{2 k}+\alpha(t, \tau)\right\} \widehat{v}(\tau) d \tau d t / 2 \pi \\
= & \overline{\left.\mathscr{F}_{t \rightarrow\left(x-J^{-1}\right.}\right)} \eta^{1 / 2}\left[\left\{D_{t}^{2 k}+\alpha\left(t, D_{t}\right)\right\} v(t)\right],
\end{aligned}
$$

where

$$
\alpha(t, \tau)=\sum_{s=0}^{2 \epsilon}\left(-t \eta^{-1 / 2}\right)^{s} c_{s}\left(\eta^{-1 / 2} \tau+j^{-1}, \eta\right) \eta^{s+k}
$$

and $\widehat{v}(\tau)=u_{j}\left(\eta^{-1 / 2} \tau+j^{-1}\right)$. It follows from (2.2) that

$$
\text { supp } \widehat{v}(\tau) \subset\left\{|\tau| \leq \eta^{1 / 2} j^{-2} / 3\right\} \equiv \omega_{0} .
$$

We choose a positive $\delta_{0}<1 / 2$ such that $\eta^{\partial_{10}}<\eta^{1 / 2} j^{-2} / 3$ with $\eta=\eta_{j}=\exp \left(j^{2} / 4 m\right)$. Since $1 \leq k \leq m-\ell-1$ it follows from (2.11) that we have for any $\varepsilon>0$ and any $0<\delta<\delta_{0}$

$$
\left|\partial_{t}^{q} \partial_{\tau}^{q^{\prime}} \alpha(t, \tau)\right| \leq C_{\varepsilon, q, q^{\prime}} \eta^{\varepsilon-(k+2)-\partial\left(q+q^{\prime}\right)} \quad \text { if } \quad|t| \leq 10 \eta^{1 / 2} .
$$


If $h_{j}(t)=h(t)$ is defined by

$$
\left\{D_{t}^{2 k}+\alpha\left(t, D_{t}\right)\right\} v(t)=h(t)
$$

then the proof of Theorem B is reduced to find some $v(t), h(t) \in \mathscr{Y}$ satisfying (2.12) and the following:

$$
\begin{aligned}
& |v(0)|>1 / 2 \text { and }|v(t)| \leq \text { Const. } \eta^{2 \delta+1 / 4} \\
& |h(t)| \leq \text { Const. } \eta^{-N} \text { on }|t| \leq \eta^{1 / 2} \\
& \|h\|_{L^{\prime}} \leq \text { Const. } \eta^{c} \text { for a } c>0 .
\end{aligned}
$$

(Here $N=N_{j}$ and $\eta=\eta_{j}$ ). In fact, we have $\widehat{f}_{j}(\xi)=\eta_{j}^{-1 / 2} e^{-i \xi / j} h\left(\xi / \eta_{j}^{1 / 2}\right)$.

Let $0 \leq \theta(t) \leq 1$ be a $C_{0}^{\infty}((-1,1))$ function such that $\theta=1$ in $|t| \leq 1 / 2$. Set $\chi_{0}(t)=\theta\left(t / 5 \eta^{1 / 2}\right)$ and $\chi_{1}(t)=\theta\left(t / 10 \eta^{1 / 2}\right)$. We are looking for a solution to

$$
\left\{D_{t}^{2 k}+\chi_{0}(t) \alpha\left(t, D_{t}\right) \chi_{1}(t)\right\} w(t)=0
$$

First we set $w_{0}(t) \equiv 1$. If $w(t)=w_{0}(t)+w_{1}(t)$ then

$$
\left\{D_{t}^{2 k}+A\left(t, D_{t}\right)\right\} w_{1}(t)=-\chi_{0} \alpha \chi_{1}(\equiv g(t))
$$

where $A\left(t, D_{t}\right)=\chi_{0}(t) \alpha\left(t, D_{t}\right) \chi_{1}(t)$. Consider this equation in the interval $I=\left(-10 \eta^{1 / 2}, 10 \eta^{1 / 2}\right)$ with the Dirichlet boundary condition

$$
D_{t}^{q} w_{1}\left( \pm 10 \eta^{1 / 2}\right)=0, q=0,1, \ldots, k-1 .
$$

It follows from (2.13) that $|(A u, u)| \leq C \eta^{\varepsilon-k-2}\|u\|^{2}$ and $\left\|D_{t}^{k} u\right\|^{2} \geq C \eta^{-k}\|u\|^{2}$. If $G_{\eta}$ denotes the Green operator for this boundary value problem then

$$
\left\|G_{\eta} f\right\|_{L^{2}(I)} \leq C \eta^{k}\|f\|_{L^{2}(I)} \text { for } f \in L^{2}(I) .
$$

Since $\left\|\chi_{1}\right\|_{L^{2}} \doteq O\left(\eta^{1 / 4}\right)$ we have $\|g\|_{L^{2}}=O\left(\eta^{1 / 4-k-2+\varepsilon}\right)$ by means of (2.13). It follows from (2.18) and (2.20) that $\left\|w_{1}\right\|_{L^{2}(I)}=O\left(\eta^{\varepsilon-7 / 4}\right)$. By (2.18) and (2.19) we have

$$
\left\|D_{t}^{k} w_{1}\right\|_{L^{2}(I)}^{2}+\left(A\left(t, D_{t}\right) w_{1}, w_{1}\right)=\left(g, w_{1}\right),
$$

so that $\left\|D_{t}^{k} w_{1}\right\|_{L^{2}(I)}=O\left(\eta^{\varepsilon-7 / 4}\right)$. If we extend $w_{1}$ outside of $I$ by $w_{1}=0$ then $w_{1} \in C_{0}^{k}$ and by the interpolation $\left\|D_{t}^{p} w_{1}\right\|_{L^{2}(I)}=O\left(\eta^{\varepsilon-7 / 4}\right)$ for $p=0, \ldots, k$. In case of $k \geq 2$, it follows from the Sobolev lemma that $\left\|w_{1}\right\|_{L^{\prime}}=O\left(\eta^{\varepsilon-7 / 4}\right)$ and $w(0)=1+O\left(\eta^{-1}\right)$. When $k=1$ it follows from (2.18) again that $\left\|D_{t}^{2} w_{1}\right\|_{L^{2}(I)}=$ $O\left(\eta^{1 / 4-3+\varepsilon}\right)$ and hence $\left\|\chi_{1} w_{1}\right\|_{L^{\prime}}=O\left(\eta^{\varepsilon-7 / 4}\right)$. After all we see $w(0)=1+O\left(\eta^{-1}\right)$. In view of (2.17) we have

$$
\left\{D_{t}^{2 k}+\chi_{0}(t) \alpha\left(t, D_{t}\right)\right\} \chi_{1}(t) w(t)=D_{t}^{2 k}\left(\chi_{1}-1\right) w\left(\equiv F_{1}(t)\right) .
$$

Note that $F_{1}(t)=0$ for $|t| \leq 3 \eta^{1 / 2}$. We set $\psi_{1}\left(D_{t}\right)=\theta\left(D_{t} \eta^{-\delta}\right)$. Then

$$
\left\{D_{t}^{2 k}+\chi_{0}(t) \alpha\left(t, D_{t}\right)\right\} \psi_{1}\left(D_{t}\right) \chi_{1}(t) w(t)=\psi_{1} F_{1}-\left[\chi_{0} \alpha, \psi_{1}\right] \chi_{1} w .
$$

Here $\psi_{1} F_{1}$ satisfies $\psi_{1} F_{1}=O\left(\eta^{-\infty}\right)$ on $|t| \leq \eta^{1 / 2}$. Set $v_{1}(t)=\psi_{1}\left(D_{t}\right) \chi_{1}(t) w(t)$. 
Then we have

$$
\left\|D_{t}^{p} v_{1}\right\|_{L^{2}}=O\left(\eta^{\delta p+1 / 4}\right), p=0,1,2, \ldots,
$$

and $v_{1}(t)=O\left(\eta^{2 \delta+1 / 4}\right)$. Furthermore, $v_{1}(0)=1+O\left(\eta^{-1 / 2}\right)$. In fact,

$$
\begin{aligned}
\left|\left(1-\psi_{1}\left(D_{t}\right)\right) \chi_{1}(t) w(t)\right| & =\left|\iint e^{i(t-s) \tau} \tau^{-2}(1-\psi(\tau))\left(-D_{s}\right)^{2}\left\{\chi_{1}(s) w(s)\right\} d s d \tau\right| \\
& \leq \text { Const. } \int_{\eta^{i} / 2}^{\infty} \tau^{-2} d \tau\left\{\int\left|D_{s}^{2} \chi_{1}\right| d s+\int\left|D_{s}^{2}\left(\chi_{1} w_{1}\right)\right| d s\right\} \\
& =\text { Const. } \eta^{-\delta-1 / 2} .
\end{aligned}
$$

If we set $g_{2} \equiv\left[\chi_{0} \alpha, \psi_{1}\right] \chi_{1} w$ then $\left\|g_{2}\right\|_{L^{2}}=O\left(\eta^{\varepsilon-2 \delta-k-7 / 4}\right)$. Let $w_{2}$ be $G_{\eta} g_{2}$, that is, a solution to

$$
\left\{D_{t}^{2 k}+A\right\} w_{2}=g_{2} \quad \text { in } \quad I
$$

with the Dirichlet boundary condition. By the similar way as for $w_{1}$ we have $\left\|w_{2}\right\|_{L^{2}(I)}=\mathrm{O}\left(\eta^{\varepsilon-2 \delta-7 / 4}\right)$. It follows from (2.21) that

$$
\left\{D_{t}^{2 k}+\chi_{0}(t) \alpha\left(t, D_{t}\right)\right\} v_{1}=\psi_{1} F_{1}-g_{2}=\psi_{1} F_{1}-\left\{D_{t}^{2 k}+\chi_{0}(t) \alpha\left(t, D_{t}\right)\right\} \chi_{1} w_{2}+F_{2},
$$

where $F_{2}=D_{t}^{2 k}\left(\chi_{1}-1\right) w_{2}$. Set $\psi_{q}\left(D_{t}\right)=\theta\left(D_{t} \eta^{-\delta} / 2^{q-1}\right)$ for $q=2,3, \ldots$ If $v_{2}=\psi_{2}\left(D_{t}\right) \chi_{1}(t) w_{2}(t)$ then we have

$$
\left\|D_{t} v_{2}(t)\right\|_{L^{2}}=O\left(\eta^{\delta(p-2)+\varepsilon-7 / 4}\right), p=0,1, \ldots
$$

Furthermore we have

$$
\left\{D_{t}^{2 k}+\chi_{0}(t) \alpha\left(t, D_{t}\right)\right\}\left(v_{1}+v_{2}\right)=-\left[\chi_{0} \alpha, \psi_{2}\right] \chi_{1} w_{2}+\psi_{1} F_{1}+\psi_{2} F_{2}+\left(\psi_{2}-1\right) \chi_{0} \alpha v_{1} .
$$

Since $\psi_{2} \supset \supset \psi_{1}$ the last term of the right hand side equals $O\left(\eta^{-\infty}\right)$. If we set $g_{3}=\left[\chi_{0} \alpha, \psi_{2}\right] \chi_{1} w_{2}$ then $\left\|g_{3}\right\|=O\left(\eta^{2 \varepsilon-4 \delta-k-2-7 / 4}\right)$. Set $w_{3}=G_{\eta} g_{3}$ and $v_{3}=$ $\psi_{3}\left(D_{t}\right) \chi_{1} w_{3}$. Repeat this procedure $N_{j}$ times with $2^{N_{J}}<\eta_{j}^{\delta_{1}-\delta} \leq 2^{N_{J}+1}$. Setting $v=\sum_{q=1}^{N_{j}} v_{q}$ we have

$$
\begin{aligned}
& \left\{D_{t}^{2 k}+\chi_{0}(t) \alpha\left(t, D_{t}\right)\right\} v(t) \\
= & -\left[\chi_{0} \alpha, \psi_{N_{J}}\right] \chi_{1} w_{N_{s}}+\sum_{q=1}^{N_{j}} \psi_{q} F_{q}+\sum_{q=1}^{N_{j}-1}\left(\psi_{q+1}-1\right) \chi_{0} \alpha v_{q} \\
\equiv & -g_{N_{j}}(t)+\widetilde{h}_{1}(t)+\widetilde{h}_{2}(t) .
\end{aligned}
$$

By checking the preceding argument carefully it is not difficult to see that there exists a $C_{0}>0$ independent of $j$ such that

$$
\begin{aligned}
& \left\|D_{t}^{p} v_{q}\right\|_{L^{2}} \leq C_{0}^{q} \eta^{p \delta+1 / 4+(\varepsilon-2 \delta-2)(q-1)} \\
& \text { for } p=0,1, \ldots, 2 k+2 \text { and } q=1,2, \ldots
\end{aligned}
$$

Since $C_{0} \ll \eta=\eta_{j}$ for a sufficiently large $j$ we see (2.15) by means of the Sobolev lemma. By the similar way as for $(2.25)$ we have 


$$
\left\|g_{N_{J}}\right\|_{L^{\prime}} \leq C\left\|\left(1+D_{t}^{2}\right) g_{N_{\jmath}}\right\|_{L^{2}} \leq C C_{1}^{N_{\prime}} \eta^{2 \delta+1 / 4-k+(\varepsilon-2 \delta-2) N_{J}}
$$

for $C, C_{1}>0$ independent of $j$. Similarly, we see that

$$
\begin{aligned}
& \left|\psi_{q} F_{q}(t)\right| \leq C_{2}^{q} \eta^{-N_{\jmath}+(\varepsilon-2 \delta-2) q} \text { on }|t| \leq \eta^{1 / 2} \\
& \left\|\left(\psi_{q+1}-1\right) \chi_{0} \alpha v_{q}\right\|_{L^{\prime}} \leq C_{3}^{q} \eta^{-N_{J}+(\varepsilon-2 \delta-2) q}
\end{aligned}
$$

for $C_{2}, C_{3}>0$ independent of $j$. Since $h=-g_{N_{1}}+\widetilde{h}_{1}+\widetilde{h}_{2}$ on $|t| \leq \eta^{1 / 2}$, from the above three estimates we obtain $|h(t)| \leq \eta^{-N_{\prime}}$ on $|t| \leq \eta^{1 / 2}$ if $j$ is large enough. In view of (2.14), it follows from (2.25) that $\|h\|_{L^{\prime}} \leq$ Const. $\eta^{(2 k+2) d+1 / 4}$ because $\left\|\left(1+D_{t}^{2}\right) v\right\|_{L^{2}}=O\left(\eta^{2 \delta+1}\right)$ and $\left(1+D_{t}^{2}\right) \alpha\left(t, D_{t}\right)\left(1+D_{t}^{2}\right)^{-1}$ is $L^{2}$ bounded. Hence (2.16) is fulfilled. Now the proof of Theorem $B$ is completed.

Remark. In the same way it is possible to prove the non-micro-hypoellipticity of the operator $D_{x}+i g_{k}(x) D_{y}^{m}$ with $m \geq 2 k+2$ (cf. (0.3) of [5]).

\section{References}

[1] Fediù, V.S., On a criterion for hypoellipticity, Math. USSR SB., 14 (1971), 15-45.

[2] Morimoto, Y., On the hypoellipticity for infinitely degenerate semi-elliptic operators, J. Math. Soc. Japan, 30 (1978), 327-358.

[3] Morioka, T., Hypoellipticity for semi-elliptic operators which degenerate on hypersurface, Osaka J. Math., 28 (1991), 563-578.

[4] Some remarks on micro-hypoelliptic operators of infinitely degenerate type, Publ. RIMS, Kyoto Univ., 28 (1992), 129-138.

[5] Parenti, C. and Rodino. L. Examples of hypoelliptic operators which are not micro-hypoelliptic, Boll. Un. Mat., 17-B (1980), 390-409. 